UB-ECM-PF 94/17

\title{
On the instability of the vacuum in multidimensional scalar theories
}

\author{
E. Elizalde*, K. Kirsten ${ }^{\dagger}$ and Yu. Kubyshin ${ }^{\ddagger}$ \\ Departament d'ECM, Facultat de Física \\ Universitat de Barcelona, Av. Diagonal 647, 08028 Barcelona \\ Catalonia, Spain
}

October 14, 1994

\begin{abstract}
The 1-loop effective potential in a scalar theory with quartic interaction on the space $M^{4} \times T^{n}$ for $n=2$ is calculated and is shown to be unbounded from below. This is an indication of a possible instability of the vacuum of the $\lambda \phi^{4}$ model on $M^{4}$, when it is regarded as a low energy sector of the theory obtained by dimensional reduction of the original six-dimensional one. The issue of stability for other values of the number $n$ of extra dimensions is also discussed.
\end{abstract}

${ }^{*}$ And Center for Advanced Studies, CSIC, Camí de Santa Bàrbara, 17300 Blanes, Spain. E-mail address: eli@zeta.ecm.ub.es

${ }^{\dagger}$ Alexander von Humboldt-fellow. E-mail address: klaus@zeta.ecm.ub.es

${ }^{\ddagger}$ On leave of absence from Nuclear Physics Institute, Moscow State University, 117234 Moscow, Russia.

${ }^{\S}$ E-mail address: kubyshin@ecm.ub.es 


\section{Introduction}

It is well known that the quantum properties of models in quantum field theory strongly depend on the topology of the spacetime on which they are formulated and on the boundary conditions imposed on it. An example of such manifestations is the influence that non-trivial spacetime topology has on the behaviour of the effective potential [1], [2], and, in particular, the issue of topological mass generation [3], [4]. The Casimir effect [5] is another phenomenon of this type (for a report, see [6]).

In this article we consider a scalar model with quartic interaction $\lambda \phi^{4}$ defined on $M^{4} \times T^{n}$, which is regarded as a laboratory for studying certain quantum properties of field theories in multidimensional space-times. The latter are viewed as important candidates for unification of fundamental interactions (see, for example, [7] and [8] for reviews). One of the attractive features of this approach is that it provides a natural mechanism for the appearence of scalar Higgs fields as extra dimensional components of multidimensional gauge fields with the Higgs potential being a part of the multidimensional non-abelian gauge action [9]. Much work has been done on the analysis of symmetries and structure of Kaluza-Klein type theories and on the possibility of obtaining the Standard Model of Grand Unified Theories by dimensional reduction (see [10], [11] for reviews). Various issues of quantum properties of multidimensional theories were studied in [12].

It is known that a multidimensional model can be re-formulated as a model in four dimensions with an infinite number of fields or modes. The sector of the zero mode with the lightest mass usually coincides with the model in four dimensions with the same type of fields and interactions as the initial multidimensional one, that is the $\lambda \phi^{4}$ theory on $M^{4}$ in our case. In this paper we consider the 1-loop effective potential of the zero mode field calculated in the complete theory with all modes. As it is well known by now, the effective potential is a very important tool in considering stability questions and the phenomenon of symmetry breaking (see for example [13]). We will be interested specifically in the case $n=2$, though the analysis of the asymptotics of the potential for large $\phi$ will be carried out for arbitrary $n$. It is obvious, that since the theory considered for $n \geq 1$ is non-renormalizable, renormalizations of the mass and the quartic coupling are not enough for eliminating all ultraviolet divergences. One needs to impose additional conditions to obtain the renormalized effective potential, and we will suggest a prescription for doing this at the 1-loop level. This prescription will guarantee that in the limit of weak fields the potential of the complete theory approaches the potential of the corresponding four dimensional theory, i.e. the Coleman-Weinberg effective potential [14. Namely, when $\lambda \phi^{2} L / m \ll 1$, where $L$ is the characteristic size of the torus, these two potentials differ by higher powers of $\phi$ only. Thus, the physics described by these two potentials will be the same in such a limit. For stronger fields the effects due to the non-trivial spacetime topology become significant and the behaviour of the effective potentials turns out to be quite different. It is worth mentioning here that analogous renormalization prescriptions for the Green functions imply that physical observables, like for example the cross sections, of the $\lambda \phi^{4}$ theory on $M^{4}$ coincide with those of the corresponding multidimensional model in the low-energy limit 15. 
To see the effect that the extra dimensions with non-trivial topology have on the effective potential, we restrict our study here to a simple scalar model, calculate the effective potential of the zero mode in the 1-loop approximation and compare it with the known result of Coleman and Weinberg for the usual four-dimensional $\lambda \phi^{4}$ model [14]. The model might be relevant for the description of the Higgs sector of some Kaluza-Klein type extensions of the Standard Model and in spite of its simplicity it features some important properties of Kaluza-Klein models.

We will do our calculations for the case of periodic and antiperiodic boundary conditions for the scalar field on $T^{2}$ and will also analyze the mixed case of the field periodic in one dimension and antiperiodic in the other, taking into account also interactions between the different types of scalar fields.

We adopt the point of view that the size $L$ of the space of extra dimensions (the twodimensional torus in our case) can take values in a wide range, instead of choosing it of the order of the Planck length, as it is suggested by most of spontaneous compactification solutions. The estimates, carried out in [16], give the bound on the present value $L^{-1}<10^{6}$ $\mathrm{GeV}$. Other motivations are related to results of papers [17] and suggest that $L^{-1}$ should be of the order of the supersymmetry breaking scale $M_{S U S Y}$. Thus, for a class of string models with orbifold compactification the bounds on $L$ following from high-energy experiments give $L^{-1} \sim 1 \mathrm{TeV}[18$. The parameter $L$ in our analysis plays a role similar to that of the inverse temperature $T^{-1}$ in models of non-zero temperature. We would like to mention that calculations of the effective potential in theories with non-zero temperature, when the spacetime can be effectively taken as $M^{3} \times S^{1}$ and $L=T^{-1}$ is the radius of the circle $S^{1}$, can be found in 119 .

One important remark is in order. In our calculations gravity is considered classically as a non-trivial spacetime background. This makes sense if we limit ourselves to the scalar field sector only and assume that the geometry of the space of extra dimensions and its size $L$ are classical variables and are determined by some additional mechanism. Results of ref. [20] show that in a more general theory including gravity, where $L$ is considered as a dynamical variable, contributions from gravitons are significant if $L$ is of the order of the Planck length. Then the total effective potential should include the contribution from scalars, we calculate here, and the graviton part, calculated in [20], and can be used for the analysis of the spontaneous compactification solutions of the theory of gravity coupled to scalars. To be relevant to the cosmology of the early Universe the effective potential should be further modified to include contributions due to non-zero temperature. The finite temperature graviton effective potential on the spacetime $M^{4} \times T^{n}$ was calculated by Appelquist et al. 21] and temperature corrections were found to be quite important (see also [22]). The finite temperature effective potential for the free scalar theory on the same spacetime was studied in [23] and the stability of the total effective potential was analyzed in 24]. The calculation of temperature corrections to the scalar effective potential in the model with interaction which is under consideration in this article and the analysis of spontaneous compactification solutions and their stability for that case would be of great interest.

The paper is organized as follows. In Sect. 2 we describe the model and discuss its 
unrenormalized effective potential. In Sect. 3 the renormalized effective potential on the spacetime $M^{4} \times T^{2}$ with periodic boundary conditions for the scalar field is calculated using zeta-function techniques and its properties for weak and strong fields are studied. Representations for the zeta-functions used for these calculations are given in Appendix A. Sect. 4 is devoted to an extension of our results to the case of antiperiodic and mixed boundary conditions for the scalar field including interactions between the different types of scalar fields. The asymptotics of the potential for large $\phi$ and its boundedness or unboundedness from below for more general spaces $M^{4} \times T^{n}$ are analyzed in Sect. 5 . Finally, Sect. 6 contains the discussion of the results. Some auxilliary results on the Epstein-type zeta-functions and formulas of the 1-loop contributions for the renormalized effective potential are presented in Appendices A-C.

\section{Description of the model and the effective potential}

Let us consider a one component scalar field on the 6-dimensional manifold $E=M^{4} \times T^{2}$, where $M^{4}$ is Minkowski space-time and $T^{2}$ is the two-dimensional torus with circumferences $L_{1}$ and $L_{2}$. In spite of its simplicity this model captures some interesting features of both classical and quantum properties of multidimensional theories. The classical action is given by

$$
S=\int_{E} d^{4} x d^{2} y\left[\frac{1}{2}\left(\frac{\partial \hat{\phi}(x, y)}{\partial x^{\mu}}\right)^{2}+\frac{1}{2}\left(\frac{\partial \hat{\phi}(x, y)}{\partial y^{i}}\right)^{2}-\frac{1}{2} m_{0}^{2} \hat{\phi}^{2}(x, y)-\frac{\hat{\lambda}}{4 !} \hat{\phi}^{4}(x, y)\right],
$$

where $x^{\mu}, \mu=0,1,2,3$, are the coordinates on $M^{4}$ and $y^{1}$ and $y^{2}$ are the coordinates on $T^{2}, 0 \leq y^{1}<L_{1}, 0 \leq y^{2}<L_{2}$. To re-interpret this model in four-dimensional terms we make the Fourier expansion of the field $\hat{\phi}(x, y)$,

$$
\hat{\phi}(x, y)=\sum_{N} \phi_{N}(x) Y_{N}(y)
$$

where $N=\left(l_{1}, l_{2}\right),-\infty<l_{i}<\infty$ and $Y_{N}(y)$ are the eigenfunctions of the Laplace operator on the internal space

$$
Y_{\left(l_{1}, l_{2}\right)}=\frac{1}{\sqrt{L_{1} L_{2}}} \exp \left[2 \pi i\left(\frac{l_{1} y^{1}}{L_{1}}+\frac{l_{2} y^{2}}{L_{2}}\right)\right] .
$$

Substituting this expansion into the action and integrating over $y$, one obtains

$$
\begin{aligned}
S= & \int_{M^{4}} d^{4} x\left\{\frac{1}{2}\left(\frac{\partial \phi_{(0,0)}(x)}{\partial x^{\mu}}\right)^{2}-\frac{1}{2} m_{0}^{2} \phi_{(0,0)}^{2}(x)-\frac{\lambda}{4 !} \phi_{(0,0)}^{4}(x)\right. \\
& +\sum_{N>0}\left[\frac{\partial \phi_{N}^{*}(x)}{\partial x^{\mu}} \frac{\partial \phi_{N}(x)}{\partial x_{\mu}}-M_{N}^{2} \phi_{N}^{*}(x) \phi_{N}(x)\right] \\
& \left.-\frac{\lambda}{2} \phi_{(0,0)}^{2}(x) \sum_{N>0} \phi_{N}^{*}(x) \phi_{N}(x)\right\}-S_{i n t}^{\prime},
\end{aligned}
$$


where the four-dimensional coupling constant $\lambda$ is related to the multidimensional one $\hat{\lambda}$ by $\lambda=\hat{\lambda} / \operatorname{volume}\left(T^{2}\right)$. In eq. (幽 $S_{\text {int }}^{\prime}$ contains all terms of the third and fourth powers in $\phi_{N}$ with $N^{2}>0$. We see that the model includes one real scalar field $\phi_{(0,0)}$ describing a light particle of mass $m_{0}$, and an infinite set ("tower") of massive complex fields $\phi_{N}(x)$ corresponding to heavy particles, or pyrgons, of mass given by

$$
M_{N}^{2}=m_{0}^{2}+l_{1}^{2} M_{1}^{2}+l_{2}^{2} M_{2}^{2}
$$

where $M_{i}=2 \pi / L_{i}$.

In the spacetime considered here one may assume a constant classical background field $\hat{\phi}_{c l}$, and the quantum fluctuations $\varphi=\hat{\phi}-\hat{\phi}_{c l}$ around the background field in the linear approximation satisfy the equation

$$
\left(-\Delta+\Phi^{2}\right) \varphi=0
$$

with the effective mass $\Phi^{2}=m_{0}^{2}+(1 / 2) \hat{\lambda} \hat{\phi}_{c l}^{2}=m_{0}^{2}+(1 / 2) \lambda \phi^{2}$, where we have expressed the effective mass through the four dimensional quantities. The effective potential for the field $\phi$ including one-loop quantum effects is then given by the function

$$
V_{E}(\phi)=\frac{1}{2} m_{B}^{2} \phi^{2}+\frac{1}{4 !} \lambda_{B} \phi^{4}+V_{2} V\left(\Phi^{2}\right)
$$

where $m_{B}$ and $\lambda_{B}$ are the bare mass and coupling constant and the quantum corrections amount to

$$
\left(V_{4} V_{2}\right) V\left(\Phi^{2}\right)=\frac{1}{2} \ln \operatorname{det}\left(\frac{-\Delta+\Phi^{2}}{\kappa^{2}}\right),
$$

which is the functional determinant arising from the integration over the quantum fluctuations, $V_{4}$ and $V_{2}$ are the volumes of $M^{4}$ and $T^{2}$ respectively and $\kappa$ is a scale which arises in this formalism. General methods for the calculation of the effective potential were developed in [14], 25]; for calculations of the effective potential in theories in spaces with nontrivial topologies and for recent results in the Standard Model at non-zero temperature see [26] and 27], respectively.

For the calculation of the functional determinant, eq. (8), we will use the zeta-function prescription [28]. In this scheme

$$
V_{2} V\left(\Phi^{2}\right)=-\frac{1}{2}\left[\zeta\left(0 ; M^{4} \times T^{2}\right) \ln \kappa^{2}+\zeta^{\prime}\left(0 ; M^{4} \times T^{2}\right)\right]
$$

where the prime denotes differentiation with respect to $s$, see eq. (11). $\zeta\left(s ; M^{4} \times T^{2}\right)$ is the zeta function associated with the operator (6) with periodic boundary conditions for the field $\varphi$. This means that for $\Re e s>3$,

$$
\zeta\left(s ; M^{4} \times T^{2}\right)=\frac{1}{(2 \pi)^{4}} \sum_{l_{1}, l_{2}=-\infty}^{\infty} \int d^{4} k\left[\left(\frac{2 \pi l_{1}}{L_{1}}\right)^{2}+\left(\frac{2 \pi l_{2}}{L_{2}}\right)^{2}+k^{2}+\Phi^{2}\right]^{-s}
$$


or, performing the $k$-integration,

$$
\zeta\left(s ; M^{4} \times T^{2}\right)=\frac{\pi^{2}}{L^{4}}\left(\frac{L}{2 \pi}\right)^{2 s} \frac{\Gamma(s-2)}{\Gamma(s)} Z_{2}^{v^{2}}\left(s-2 ; w_{1}, w_{2}\right),
$$

where we have introduced the dimensionless parameters $v^{2}=(L \Phi / 2 \pi)^{2}$ and $w_{i}=\left(L / L_{i}\right)^{2}$ ( $L$ is an arbitrary length parameter). The generalized Epstein zeta-function $Z_{2}^{v^{2}}\left(\nu ; w_{1}, w_{2}\right)$ has the representation

$$
Z_{2}^{v^{2}}\left(\nu ; w_{1}, w_{2}\right)=\sum_{l_{1}, l_{2}=-\infty}^{\infty}\left[w_{1} l_{1}^{2}+w_{2} l_{2}^{2}+v^{2}\right]^{-\nu}
$$

valid for $\Re e \nu>1$, and is very well studied by now [2, 4. We have listed its essential properties in Appendix A, indicating very briefly their derivation. Comparing with eq. (A.1), we use here the notation $Z_{2}^{v^{2}}\left(\nu ; w_{1}, w_{2}\right)=Z_{2}^{v^{2}}\left(\nu ; w_{1}, w_{2} ; 0,0\right)$. From (9), the quantum potential is found to be

$$
\begin{aligned}
V_{2} V\left(\Phi^{2}\right)= & -\frac{1}{4} \frac{\pi^{2}}{L^{4}}\left\{Z_{2}^{\prime v^{2}}\left(-2 ; w_{1}, w_{2}\right)-\frac{\pi}{3} v^{6} \frac{L_{1} L_{2}}{L^{2}}\left[2 \ln \left(\frac{L \kappa}{2 \pi}\right)+\frac{3}{2}\right]\right\} \\
= & \frac{\pi^{3} L_{1} L_{2}}{12 L^{6}} v^{6}\left[\frac{11}{6}-\ln \left(\frac{\Phi}{\kappa}\right)^{2}\right] \\
& -\frac{L_{1} L_{2}}{L^{6}} v^{3} \sum_{l_{1}, l_{2}=-\infty}^{\prime}\left[\frac{l_{1}^{2}}{w_{1}}+\frac{l_{2}^{2}}{w_{2}}\right]^{-\frac{3}{2}} K_{3}\left(2 \pi v\left[\frac{l_{1}^{2}}{w_{1}}+\frac{l_{2}^{2}}{w_{2}}\right]^{\frac{1}{2}}\right),
\end{aligned}
$$

where in the last equality eq. (A.4) has been used. Eq. (13) represents the non-renormalized one-loop contribution to the effective potential of the theory. As one can easily check, in spite of the presence of the parameter $L$ in eq. (13), the potential does not depend on it actually. The dependence on $\kappa$ will disappear after renormalization.

\section{Renormalization of the one-loop effective potential}

In the previous section we have derived the regularized one-loop contribution to the effective potential $V\left(\Phi^{2}\right)$ for a scalar theory defined on the manifold $E=M^{4} \times T^{2}$. In accordance with the discussion in the Introduction, we need to consider a potential of the following general form

$$
\begin{aligned}
V_{E}(\phi)= & \frac{1}{2} m_{0}^{2} \phi^{2}+\frac{1}{4 !} \lambda \phi^{4} \\
& +A+\frac{1}{2} B \phi^{2}+\frac{1}{4 !} C \phi^{4}+\frac{1}{6 !} D \phi^{6}+V_{2} V\left(\Phi^{2}\right),
\end{aligned}
$$

where $m_{0}$ and $\lambda$ are the renormalized mass and coupling constant, respectively, and the bare parameters are understood to be defined as $m_{B}^{2}=m_{0}^{2}+B, \lambda_{B}=\lambda+C$. The constant $D$ subtracts the additional divergences arising due to the six-dimensional nature of the 
theory. For the $\lambda \phi^{4}$ - model on $M^{4}$ to be a low-energy limit of our multidimensional theory, we impose the following renormalization conditions:

$$
\begin{aligned}
\left.V_{E}(\phi)\right|_{\phi=0} & =0=\left.V_{M^{4}}(\phi)\right|_{\phi=0}, \\
\left.\frac{\partial^{2} V_{E}(\phi)}{\partial \phi^{2}}\right|_{\phi=0} & =m_{0}^{2}=\left.\frac{\partial^{2} V_{M^{4}}(\phi)}{\partial \phi^{2}}\right|_{\phi=0}, \\
\left.\frac{\partial^{4} V_{E}(\phi)}{\partial \phi^{4}}\right|_{\phi=\mu} & =\lambda=\left.\frac{\partial^{4} V_{M^{4}}(\phi)}{\partial \phi^{4}}\right|_{\phi=\mu}, \\
\left.\frac{\partial^{6} V_{E}(\phi)}{\partial \phi^{6}}\right|_{\phi=\mu} & =\left.\frac{\partial^{6} V_{M^{4}}(\phi)}{\partial \phi^{6}}\right|_{\phi=\mu}+h
\end{aligned}
$$

where, for the sake of generality, we have introduced in the last equation the additional coupling of the $\phi^{6}$-vertex, that appears in principle because of the non-renormalizability of the theory. $V_{M^{4}}(\phi)$ is the Coleman-Weinberg potential in the four dimensional Minkowski spacetime before renormalization. It reads [14

$$
V_{M^{4}}(\phi)=\frac{1}{2} m_{0}^{2} \phi^{2}+\frac{1}{4 !} \lambda \phi^{4}+\frac{1}{64 \pi^{2}} \Phi^{4}\left[\ln \left(\frac{\Phi}{\kappa}\right)^{2}-\frac{3}{2}\right]+A^{\prime}+\frac{1}{2} B^{\prime} \phi^{2}+\frac{1}{4 !} C^{\prime} \phi^{4}
$$

with $A^{\prime}, B^{\prime}, C^{\prime}$ being determined by the conditions (15)- (17). For zero mass, $m_{0}^{2}=0$, one has to choose $\mu \neq 0$. As we will see in what follows, it is useful to consider the function

$$
\begin{aligned}
H\left(\Phi^{2} ; u_{1}, u_{2}\right)=-\frac{\Phi^{3} L_{1} L_{2}}{8 \pi^{3} L^{3}} \sum_{l_{1}, l_{2}=-\infty}^{\infty} ' \exp \left\{2 \pi i\left[l_{1} u_{1}+l_{2} u_{2}\right]\right\} \\
{\left[\frac{l_{1}^{2}}{w_{1}}+\frac{l_{2}^{2}}{w_{2}}\right]^{-\frac{3}{2}} K_{3}\left(L \Phi\left[\frac{l_{1}^{2}}{w_{1}}+\frac{l_{2}^{2}}{w_{2}}\right]^{\frac{1}{2}}\right), }
\end{aligned}
$$

where the phase factor has been introduced with regard to the different boundary conditions treated later on. For the moment we only need $H\left(\Phi^{2}\right):=H\left(\Phi^{2} ; 0,0\right)$.

Denoting by $H^{(n)}\left(\Phi^{2}\right)$ the $n$-th derivative with respect to $\Phi^{2}$, eqs. (15)-(18) determine the counterterms to be

$$
\begin{aligned}
A\left(m_{0}^{2}\right)= & -H\left(m_{0}^{2}\right)-\frac{L_{1} L_{2}}{768 \pi^{3}} m_{0}^{6}\left[\frac{11}{6}-\ln \left(\frac{m_{0}}{\kappa}\right)^{2}\right], \\
B\left(m_{0}^{2}\right)= & -\lambda H^{(1)}\left(m_{0}^{2}\right)-\frac{\lambda L_{1} L_{2}}{768 \pi^{3}} 3 m_{0}^{4}\left[\frac{3}{2}-\ln \left(\frac{m_{0}}{\kappa}\right)^{2}\right], \\
C= & C^{(1)}\left(m_{0}^{2}, \lambda\right) \\
& -\frac{\mu^{2}}{32 \pi^{2}}\left\{\frac{15}{2} \frac{\lambda^{3}}{\Phi_{\mu}^{2}}-\frac{45}{2} \frac{\lambda^{4} \mu^{2}}{\Phi_{\mu}^{4}}-\frac{15 \lambda^{5} \mu^{4}}{\Phi_{\mu}^{6}}-3 \frac{\lambda^{6} \mu^{6}}{\Phi_{\mu}^{8}}\right\} \\
& -\frac{1}{2} h \mu^{2}, \\
D= & D^{(1)}\left(m_{0}^{2}, \lambda\right)+\frac{15 \lambda^{3}}{32 \pi^{2} \Phi_{\mu}^{2}}-\frac{45 \lambda^{4} \mu^{2}}{32 \pi^{2} \Phi_{\mu}^{4}}
\end{aligned}
$$




$$
+\frac{30 \lambda^{5} \mu^{4}}{32 \pi^{2} \Phi_{\mu}^{6}}-\frac{6 \lambda^{6} \mu^{6}}{32 \pi^{2} \Phi_{\mu}^{8}}+h
$$

with $\Phi_{\mu}^{2}=m_{0}^{2}+\frac{1}{2} \lambda \mu^{2}$. Here $C^{(1)}\left(m_{0}^{2}, \lambda\right)$ and $D^{(1)}\left(m_{0}^{2}, \lambda\right)$ denote the contribution of the one-loop quantum correction $V_{2} V\left(\Phi^{2}\right)$ to the counterterms. Explicitly they read

$$
\begin{aligned}
C^{(1)}\left(m_{0}^{2}, \lambda\right)= & -3 \lambda^{2} H^{(2)}\left(\Phi_{\mu}^{2}\right)+\frac{3}{2} \lambda^{3} \mu^{2} H^{(3)}\left(\Phi_{\mu}^{2}\right)+\frac{43}{2} \lambda^{4} \mu^{4} H^{(4)}\left(\Phi_{\mu}^{2}\right) \\
& +\frac{15}{2} \lambda^{5} \mu^{6} H^{(5)}\left(\Phi_{\mu}^{2}\right)+\frac{1}{2} \lambda^{6} \mu^{8} H^{(6)}\left(\Phi_{\mu}^{2}\right) \\
& +\frac{L_{1} L_{2}}{768 \pi^{3}}\left\{18 \lambda^{2} m_{0}^{2} \ln \left(\frac{\Phi_{\mu}}{\kappa}\right)^{2}-129 \frac{\lambda^{4} \mu^{4}}{\Phi_{\mu}^{2}}-18 \lambda^{2} \Phi_{\mu}^{2}+\frac{45 \lambda^{5} \mu^{6}}{\Phi_{\mu}^{4}}-\frac{6 \lambda^{6} \mu^{8}}{\Phi_{\mu}^{6}}\right\}, \\
D^{(1)}\left(m_{0}^{2}, \lambda\right)= & -15 \lambda^{3} H^{(3)}\left(\Phi_{\mu}^{2}\right)-45 \lambda^{4} \mu^{2} H^{(4)}\left(\Phi_{\mu}^{2}\right)-15 \lambda^{5} \mu^{4} H^{(5)}\left(\Phi_{\mu}^{2}\right)-\lambda^{6} \mu^{6} H^{(6)}\left(\Phi_{\mu}^{2}\right) \\
+ & \frac{90 \lambda^{3} L_{1} L_{2}}{768 \pi^{3}} \ln \left(\frac{\Phi_{\mu}}{\kappa}\right)^{2}+\frac{270 \lambda^{4} \mu^{2} L_{1} L_{2}}{768 \pi^{3} \Phi_{\mu}^{2}}-\frac{90 \lambda^{5} \mu^{4} L_{1} L_{2}}{768 \pi^{3} \Phi_{\mu}^{4}}+\frac{12 \lambda^{6} \mu^{6} L_{1} L_{2}}{768 \pi^{3} \Phi_{\mu}^{6}} .
\end{aligned}
$$

Use of eqs. (21)-(24) in eq. (14) yields the renormalized effective potential. As we will see, the dependence on the arbitrary scale $\kappa$ has vanished.

For the presentation of the effective potential in the case $m_{0} \neq 0$ it is convenient to introduce dimensionless parameters. As such, we choose

$$
x=\frac{\lambda \phi^{2}}{2 m_{0}^{2}} ; \quad y=\frac{\lambda \mu^{2}}{2 m_{0}^{2}} ; g=\frac{\lambda}{64 \pi^{2}} ; \quad \nu=m_{0}^{2} L_{1} L_{2} ; \quad \xi=\frac{h m_{0}^{2}}{90 \lambda^{2}} .
$$

Furthermore let us rewrite the terms containing the McDonald (or modified Bessel) functions in the form

$$
H\left(\Phi^{2}\right)=\frac{L_{1} L_{2}}{64 \pi^{2} L^{6}} E\left(L^{2} \Phi^{2}\right)
$$

and, finally, let us introduce the following dimensionless function characterizing the effective potential:

$$
v_{E}(x)=\frac{\lambda V_{E}(\phi)}{m_{0}^{4}} .
$$

We find that

$$
v_{E}(x)=v^{(0)}(x)+v^{(1)}(x),
$$

where

$$
\begin{aligned}
v^{(0)}(x)= & x+\frac{1}{6} x^{2}-\frac{2}{3} g y x^{2}\left[\frac{15}{2} \beta-45 y \beta^{2}+60 y^{2} \beta^{3}-24 y^{3} \beta^{4}\right]-15 y \xi x^{2}+x^{3} \xi \\
& +\frac{1}{45} g x^{3}\left[15 \beta-90 y \beta^{2}+120 y^{2} \beta^{3}-48 y^{3} \beta^{4}\right]
\end{aligned}
$$

and

$$
v^{(1)}(x)=\frac{1}{12 \pi} g \nu \ln \left(\frac{m_{0}}{\Phi}\right)^{2}+\frac{1}{12 \pi} g x \nu\left[1+3 \ln \left(\frac{m_{0}}{\Phi}\right)^{2}\right]
$$




$$
\begin{aligned}
& +\frac{\nu}{72 \pi} g x^{2}\left[18 \ln \left(\frac{\Phi_{\mu}}{\Phi}\right)^{2}+15-18 y-516 y^{2} \beta+360 y^{3} \beta^{2}-96 y^{4} \beta^{3}\right] \\
& +\frac{1}{1080 \pi} g \nu x^{3}\left[90 \ln \left(\frac{\Phi_{\mu}}{\Phi}\right)^{2}+165+540 y \beta-360 y^{2} \beta^{2}+96 y^{3} \beta^{3}\right] \\
& +\left(\frac{L_{1} L_{2}}{L^{2}}\right)\left\{-\frac{g}{\alpha^{4}} E\left(m_{0}^{2} L^{2}\right)-\frac{g x}{\alpha^{2}} E^{(1)}\left(m_{0}^{2} L^{2}\right)+\frac{g}{\alpha^{4}} E\left(z^{2}\right)\right. \\
& +\frac{1}{6} g x^{2}\left[-3 E^{(2)}\left(z_{\mu}^{2}\right)+3 y \alpha^{2} E^{(3)}\left(z_{\mu}^{2}\right)+86 y^{2} \alpha^{4} E^{(4)}\left(z_{\mu}^{2}\right)\right. \\
& \left.\quad+60 y^{3} \alpha^{6} E^{(5)}\left(z_{\mu}^{2}\right)+8 y^{4} \alpha^{8} E^{(6)}\left(z_{\mu}^{2}\right)\right] \\
& \left.-\frac{g}{90} x^{3} \alpha^{2}\left[15 E^{(3)}\left(z_{\mu}^{2}\right)+90 y \alpha^{2} E^{(4)}\left(z_{\mu}^{2}\right)+60 y^{2} \alpha^{4} E^{(5)}\left(z_{\mu}^{2}\right)+8 y^{3} \alpha^{6} E^{(6)}\left(z_{\mu}^{2}\right)\right]\right\},
\end{aligned}
$$

where we use the notations

$$
\beta=\frac{1}{1+y} ; \quad \alpha=m_{0} L=\sqrt{\nu w_{1} w_{2}} ; \quad z=L \Phi=\alpha(1+x) ; \quad z_{\mu}=L \Phi_{\mu}=\alpha(1+y) .
$$

The reason for the separation chosen in eq. (29) will become clear from the next section.

Let us now study the expression for the renormalized effective potential with $m_{0} \neq 0$. First we analyze the asymptotics of eq. (32) for large $x$. Here we restrict ourselves to the case $\mu=0$ and $L_{1}=L_{2}=L$. The leading terms are:

$$
v_{E} \approx-\frac{g \nu x^{3}}{12 \pi} \ln (c x)+\frac{g+3 \xi}{3} x^{3},
$$

where $c$ is given by: $\ln c=-11 / 6+E^{(3)}(\nu) / 6$. We see that the potential is unbounded from below for large values of the field and that such behaviour is a consequence of the sixdimensional nature of the model. Indeed, the leading asymptotics $v_{E} \sim-g \nu x^{3} \ln x / 12 \pi$ is given by the first term in eq. (13). Since the renormalization conditions (15)-(18) amount to changing terms at most $\sim x^{3}$, the asymptotics are not affected by renormalization.

As mentioned above, the representation eqs. (29)-(32) is useful for large fields and finite $\nu$. However, in order to see the limit of small fields and/or how the ColemanWeinberg potential results when extra dimensions disappear in the limit $L \rightarrow 0$, it is more convenient to use another representation. To derive it, notice that the zeta function associated with the six-dimensional operator (6), can be decomposed as follows:

$$
\zeta\left(s ; M^{4} \times T^{2}\right)=\zeta\left(s ; M^{4}\right)+\tilde{\zeta}\left(s ; M^{4} \times T^{2}\right) .
$$

Here $\zeta\left(s ; M^{4}\right)$ is the 1-loop integral on $M^{4}$ given by the term with $l_{1}=l_{2}=0$ in eq. (10). After performing the $k$-integration it is equal to

$$
\zeta\left(s ; M^{4}\right)=\frac{1}{16 \pi^{2}(1-s)(2-s)}\left(\Phi^{2}\right)^{2-s} .
$$

$\tilde{\zeta}\left(s ; M^{4} \times T^{2}\right)$ is the rest of the sum (10) and contains contributions due to the extra dimensions. 
Maintaining the decomposition corresponding to (34) at each step, we perform the renormalization as before and get the result (for $m_{0} \neq 0$ ):

$$
v_{E}(x)=v_{M^{4}}(x)+\xi x^{3}-15 \xi y x^{2}+\Delta v_{1}(x)+\Delta v_{2}(x)+\Delta v_{3}(x) .
$$

Here

$$
\begin{aligned}
v_{M^{4}}(x)= & x+\frac{x^{2}}{6}-g x-\frac{3}{2} g x^{2}+g(1+x)^{2} \ln (1+x) \\
& -g x^{2} \ln (1+y)-\frac{4}{3} g x^{2} y \frac{3+2 y}{(1+y)^{2}}
\end{aligned}
$$

is the part corresponding to the renormalized one-loop Coleman-Weinberg potential $V_{M^{4}}(\phi)$ (with non-zero mass) through the relation $v_{M^{4}}\left(\lambda \phi^{2} /\left(2 m_{0}^{2}\right)\right)=\left(\lambda / M_{0}^{4}\right) V_{M^{4}}(\phi)$. The term $\Delta v_{1}(x)$ is equal to

$$
\begin{aligned}
\Delta v_{1}(x) & =g \sum_{l_{1}, l_{2}=-\infty}^{\infty},\left[\frac{1}{\nu^{2}}\left(l_{1}^{2} w_{1}+l_{2}^{2} w_{2}+\nu(1+x)\right)^{2} \ln \frac{l_{1}^{2} w_{1}+l_{2}^{2} w_{2}+\nu(1+x)}{l_{1}^{2} w_{1}+l_{2}^{2} w_{2}+\nu}\right. \\
& \left.-\frac{x}{\nu}\left(l_{1}^{2} w_{1}+l_{2}^{2} w_{2}+\nu\right)-\frac{3}{2} x^{2}-\frac{1}{3} \frac{x^{3} \nu}{\left(l_{1}^{2} w_{1}+l_{2}^{2} w_{2}+\nu\right)}\right] .
\end{aligned}
$$

The terms $\Delta v_{2}(x)$ and $\Delta v_{3}(x)$ are given in Appendix C. As one can see they vanish when $y=0$. The analogous representation for the case $m_{0}=0$ is presented in Appendix $\mathrm{C}$ too. Using these formulas we obtain the behaviour of the potential for small $x$ and small $\nu$.

We first consider the case $m_{0} \neq 0$ in more detail and for the sake of simplicity choose $\mu=0$. Then $\Delta v_{2}=\Delta v_{3}=0$. For $x \nu \ll 1$

$$
\Delta v_{1}=-\frac{g}{12} \alpha^{4} x^{4} \sum_{l_{1}, l_{2}=-\infty}^{\infty} \frac{1}{\left(w_{1} l_{1}^{2}+w_{2} l_{2}^{2}+\nu\right)^{2}}+\frac{1}{\alpha^{4}} \mathcal{O}\left(\nu^{5} x^{5}\right)
$$

and we obtain

$$
v_{E}=v_{M^{4}}(x)+\xi x^{3}-\frac{g}{12} \alpha^{4} x^{4} Z_{2}^{\alpha^{2}}\left(2 ; w_{1}, w_{2}\right)+\mathcal{O}\left(x^{5}\right) .
$$

From the formula above we see that, due to the renormalization conditions imposed, the effective potential $V_{E}$ gives the values of $m_{0}$ and $\lambda$ at the subtraction point $\phi_{0}=0$. Moreover, these conditions also assure that the contributions of the heavy modes are of the order $\phi_{0}^{8}$ for small fields, namely when $x \nu \ll 1$ or $\phi_{0}^{2} \ll 2 /\left(\lambda L^{2}\right)$.

On the other hand, from eq. (39) we see that, for any fixed $x$ and $\nu \ll 1$ (we assume that $w_{1}$ and $w_{2}$ are fixed), we get

$$
v_{E}(x)=v_{M^{4}}+\xi x^{3}-\frac{g \nu^{2} w_{1}^{2} w_{2}^{2} x^{4}}{12} Z_{2}\left(2 ; w_{1}, w_{2}\right)+\mathcal{O}\left(\nu^{3}\right)
$$

where

$$
Z_{2}\left(s ; w_{1}, w_{2}\right)=\sum_{l_{1}, l_{2}=-\infty}^{\infty} \frac{1}{\left(w_{1} l_{1}^{2}+w_{2} l_{2}^{2}\right)^{s}}
$$


It is clear that for $\xi=0$ the four-dimensional Coleman-Weinberg potential is recovered in the limit $L \rightarrow 0$, i.e. when the space of extra dimensions shrinks down to zero.

In the massless case, $m_{0}^{2}=0$, using the expansion of the Epstein-type zeta function for small values of the field, see Appendix A, eq. (A.7), we find

$$
\begin{aligned}
& V_{2} V\left(\Phi^{2}\right)=-\frac{1}{2} \frac{\pi^{2}}{L^{4}} \times \\
& \left\{-\frac{1}{2} v^{4} \ln v^{2}+\frac{1}{2} Z_{2}^{\prime}\left(-2 ; w_{1}, w_{2}\right)+v^{2} Z_{2}^{\prime}\left(-1 ; w_{1}, w_{2}\right)\right. \\
& \quad+\frac{1}{2} v^{4}\left[\frac{3}{2}+Z_{2}^{\prime}\left(0 ; w_{1}, w_{2}\right)\right] \\
& \left.\quad-\frac{1}{6} v^{6}\left[P P Z_{2}\left(1 ; w_{1}, w_{2}\right)+\frac{2 \pi L_{1} L_{2}}{L^{2}} \ln \left(\frac{L \kappa}{2 \pi}\right)\right]+g(v)\right\},
\end{aligned}
$$

with

$$
g(v)=\sum_{j=2}^{\infty}(-1)^{j} \frac{(j-1) !}{(j+2) !} v^{2 j+4} Z_{2}^{v^{2}}\left(j ; w_{1}, w_{2}\right) .
$$

Here, $P P Z_{2}\left(1 ; w_{1}, w_{2}\right)$ denotes the finite part of $Z_{2}\left(1 ; w_{1}, w_{2}\right)$. Without presenting once more the detailed calculations, by imposing the renormalization conditions, eqs. (15)-(18), and using this time the above representation, the final result for the renormalized effective potential reads

$$
\begin{aligned}
& V_{E}(\phi)=V_{M^{4}}(\phi) \\
& +\frac{1}{4 !} \phi^{4}\left\{-\frac{1}{2} h \mu^{2}+\frac{\lambda^{2}}{128 \pi^{2}} g^{(4)}\left(v_{\mu}\right)-\frac{\lambda^{3} L^{2} \mu^{2}}{2048 \pi^{4}} g^{(6)}\left(v_{\mu}\right)\right\} \\
& +\frac{1}{6 !} \phi^{6}\left\{h+\frac{\lambda^{3} L^{2}}{1024 \pi^{4}} g^{(6)}\left(v_{\mu}\right)\right\}-\frac{1}{2} \frac{\pi^{2}}{L^{4}} g(v) .
\end{aligned}
$$

The first line is exactly the Coleman-Weinberg result 14

$$
V_{M^{4}}(\phi)=\frac{1}{4 !} \lambda \phi^{4}+\frac{\lambda^{2}}{256 \pi^{2}} \phi^{4}\left[\ln \left(\frac{\phi}{\mu}\right)^{2}-\frac{25}{6}\right]
$$

the following terms are corrections to it, the last one being of the order $\mathcal{O}\left((L \phi)^{8}\right)$.

For the case $m_{0}=0$ the representation analogous to eqs. (36)-(38), useful for the analysis at small values of the field or small values of $t=(L \mu)^{2}$, is the following:

$$
u_{E}(z)=\frac{4 !}{\lambda \mu^{4}} V_{E}(\phi)=u_{M^{4}}(z)+\eta z^{3}-15 \eta z^{2}+\Delta u_{1}(z)+\Delta u_{2}(z)+\Delta u_{3}(z)
$$

where $z=\phi^{2} / \mu^{2}, \eta=h \mu^{2} /(30 \lambda)$ and

$$
u_{M^{4}}(z)=\frac{4 !}{\lambda \mu^{4}} V_{M^{4}}(\phi)
$$


is again the term corresponding to the Coleman-Weinberg potential (42). The terms $\Delta u_{i}(z), i=1,2,3$, are given in Appendix C.

Using the formulas of this section we have computed the behaviour of the functions characterizing the effective potential with $w_{1}=w_{2}=1$. In Fig. 1 the plots of $v_{M^{4}}(x)$ and $v_{E}$ for three values of $\nu$ and for $y=0, \xi=0$ are given. For small $x$ the curves are very close, whereas for large $x$ the asymptotic behaviour (33) is recovered. It is seen that for smaller values of $\nu$ the function $v_{E}(x)$ approaches the Coleman-Weinberg potential $v_{M^{4}}(x)$ for a wider range of $x$.

The plots of the functions $u_{M^{4}}(z)$ and $u_{E}(z)$ for $t=0.007,0.01$ and 0.015 are presented in Figs. 2 and 3. The curves are close to each other for small values of $z$ showing nontrivial minima, whereas with increasing $z$ the functions $u_{E}(z)$ deviate more and more from $u_{M^{4}}(z)$ and are unbounded from below for large $z$.

Varying $h$ and $w_{1}, w_{2}$ does not change the behaviour of $v_{E}(x)$ or $u_{E}(z)$ qualitatively. Essentially this only changes the values of the field where these functions begin to decrease.

The results of this section show, that the vacuum state $\phi=0$ of the four dimensional theory might be unstable due to the presence of the two extra dimensions of the original space-time.

\section{Antiperiodic and mixed boundary conditions on the torus}

Up to now we have only considered periodic boundary conditions for the scalar field in both directions of the torus $T^{2}$, see eqs. (2), (3). However, there are two further different types of scalar fields: antiperiodic in both directions, $\hat{\phi}\left(x, y^{1}+m L_{1}, y^{2}+n L_{2}\right)=$ $(-1)^{m+n} \hat{\phi}\left(x, y^{1}, y^{2}\right)$ and periodic in one direction and antiperiodic in the other $\hat{\phi}\left(x, y^{1}+\right.$ $\left.m L_{1}, y^{2}+n L_{2}\right)=(-1)^{m} \hat{\phi}\left(x, y^{1}, y^{2}\right)$. We study such possibilities in this section.

In considering them, we restrict ourselves to the case $L_{1}=L_{2}=L$, thus $w_{i}=1$. This kind of boundary conditions leads to the general type of Epstein zeta-functions

$$
Z_{2}^{v^{2}}\left(\nu ; w_{1}, w_{2} ; u_{1}, u_{2}\right)=\sum_{l_{1}, l_{2}=-\infty}^{\infty}\left[w_{1}\left(l_{1}-u_{1}\right)^{2}+w_{2}\left(l_{2}-u_{2}\right)^{2}+v^{2}\right]^{-\nu} .
$$

The relevant properties are summarized in Appendix A. For periodic boundary conditions we have $u_{1}=u_{2}=0$, for antiperiodic boundary conditions $u_{1}=u_{2}=1 / 2$, and, finally, for the mixed ones $u_{1}=0$ and $u_{2}=1 / 2$.

For antiperiodic and mixed boundary conditions alone, the analysis of the previous section makes no sense for the simple reason, that there is no constant classical background field with this boundary conditions apart from $\phi=0$. However, the influence might be present if one includes interactions between the different types of scalar fields [29]. Thus, as a generalization to eq. (1), we consider the action

$$
S=\int_{E} d^{4} x d^{2} y\left[\frac{1}{2}\left(\frac{\partial \hat{\phi}_{U}(x, y)}{\partial x^{\mu}}\right)^{2}+\frac{1}{2}\left(\frac{\partial \hat{\phi}_{U}(x, y)}{\partial y^{i}}\right)^{2}-\frac{1}{2} m_{U}^{2} \hat{\phi}_{U}^{2}(x, y)-\frac{\hat{\lambda}_{U}}{4 !} \hat{\phi}_{U}^{4}(x, y)\right.
$$




$$
\begin{aligned}
& +\frac{1}{2}\left(\frac{\partial \hat{\phi}_{T}(x, y)}{\partial x^{\mu}}\right)^{2}+\frac{1}{2}\left(\frac{\partial \hat{\phi}_{T}(x, y)}{\partial y^{i}}\right)^{2}-\frac{1}{2} m_{T}^{2} \hat{\phi}_{T}^{2}(x, y)-\frac{\hat{\lambda}_{T}}{4 !} \hat{\phi}_{T}^{4}(x, y) \\
& +\frac{1}{2}\left(\frac{\partial \hat{\phi}_{M}(x, y)}{\partial x^{\mu}}\right)^{2}+\frac{1}{2}\left(\frac{\partial \hat{\phi}_{M}(x, y)}{\partial y^{i}}\right)^{2}-\frac{1}{2} m_{M}^{2} \hat{\phi}_{M}^{2}(x, y)-\frac{\hat{\lambda}_{M}}{4 !} \hat{\phi}_{M}^{4}(x, y) \\
& \left.-\frac{1}{4} \hat{g}_{T U} \hat{\phi}_{T}^{2} \hat{\phi}_{U}^{2}-\frac{1}{4} \hat{g}_{M U} \hat{\phi}_{M}^{2} \hat{\phi}_{U}^{2}-\frac{1}{4} \hat{g}_{M T} \hat{\phi}_{M}^{2} \hat{\phi}_{T}^{2}\right]
\end{aligned}
$$

where we have included all possible quartic interactions invariant under the transformation $\hat{\phi}_{U, T, M} \rightarrow-\hat{\phi}_{U, T, M}$. Here, $\hat{\phi}_{U, T, M}$ denotes respectively scalar fields with periodic (untwisted), antiperiodic (twisted) and mixed boundary conditions.

Due to the above remark, the only constant classical background field $\phi$ we may introduce is in the untwisted sector. Owing to this structure, it is easily seen, that the analysis presented in the previous sections is already enough to deduce in a very simple way the effective potential for the theory (45).

The quantum potential consists now of three pieces, one coming from the twisted, one from the untwisted and one from the mixed sector. The interactions between the different types of scalar fields induce different effective masses depending on the couplings involved. In detail

$$
V_{4} V_{2} V\left(\Phi_{U}^{2}, \Phi_{T}^{2}, \Phi_{M}^{2}\right)=V_{4} V_{2}\left[V_{U}\left(\Phi_{U}^{2}\right)+V_{T}\left(\Phi_{T}^{2}\right)+V_{M}\left(\Phi_{M}^{2}\right)\right]
$$

with

$$
V_{4} V_{2} V_{i}\left(\Phi_{i}^{2}\right)=\frac{1}{2} \ln \left(-\frac{\Delta_{i}+\Phi_{i}^{2}}{\kappa^{2}}\right), \quad i=U, T, M
$$

and with

$$
\Phi_{U}^{2}=m_{U}^{2}+\frac{1}{2} \lambda_{U} \phi^{2} ; \quad \Phi_{T}^{2}=m_{T}^{2}+\frac{1}{2} g_{U T} \phi^{2} ; \quad \Phi_{M}^{2}=m_{M}^{2}+\frac{1}{2} g_{U M} \phi^{2} .
$$

Similarly to Sect. 2, the four dimensional coupling constants $\lambda_{U}, g_{U T}$ and $g_{U M}$, are related to the multidimensional ones by $\lambda_{U}=\hat{\lambda}_{U} /$ volume $\left(T^{2}\right)$, etc.

The contributions from the different sectors are calculated in the way presented in Sect. 2, the relevant formula here is (A.4). In order to express the result, we introduce

$$
V_{2} V\left(\Phi^{2} ; u_{1}, u_{2}\right)=\frac{\pi^{3} L_{1} L_{2}}{12 L^{6}} v^{6}\left[\frac{11}{6}-\ln \left(\frac{\Phi}{\kappa}\right)^{2}\right]+H\left(\Phi^{2} ; u_{1}, u_{2}\right)
$$

where as before $v^{2}=(L \Phi / 2 \pi)^{2}$ and the second term is defined by the formula (20) with $w_{1}=w_{2}=1$ (compare with the analogous result (13) for the periodic case). Then the regularized quantum potential reads

$$
V\left(M_{U}^{2}, M_{T}^{2}, M_{M}^{2}\right)=V\left(\Phi_{U}^{2} ; 0,0\right)+V\left(\Phi_{T}^{2} ; 1 / 2,1 / 2\right)+V\left(\Phi_{M}^{2} ; 0,1 / 2\right)
$$

Imposing once more the renormalization conditions (15)-(18), the counterterms are easily determined. From the previous discussion and the comments in Sect. 3, it is clear that no additional calculations are necessary. The only change being, that in eq. (21)-(24), 
the terms $A\left(m_{0}^{2}\right), B\left(m_{0}^{2}\right), C^{(1)}\left(m_{0}^{2}, \lambda\right), D^{(1)}\left(m_{0}^{2}, \lambda\right)$, have to be replaced by a sum of three terms with the replacement $m_{0}^{2} \rightarrow m_{U}^{2}, m_{T}^{2}$ and $m_{M}^{2}$, respectively $\lambda \rightarrow \lambda_{U}, g_{U T}$ and $g_{U M}$. Furthermore, for antiperiodic respectively mixed boundary conditions, $H\left(\Phi^{2} ; 0,0\right)$ has to be replaced by $H\left(\Phi^{2} ; 1 / 2,1 / 2\right)$, respectively by $H\left(\Phi^{2} ; 1 / 2,0\right)$. In the same way, the effective potential is given by eq. (29), once $v^{(1)}\left(m_{0}^{2}, \lambda\right)$, given by eq. (31), is replaced by the sum of the three contributions as described above. To have the $\lambda \phi^{4}$ - model in the limit $L \rightarrow 0$ we take the renormalized constants $\lambda_{U}=\lambda, m_{U}=m_{0}$. Furthermore $g_{U T}, g_{U M}$ respectively $m_{T}, m_{M}$ are the renormalized couplings of the interactions between the fields respectively masses of the fields with different boundary conditions, which we assume to be all positive.

Representations similar to (36)-(38) in this case are (for $m_{0}^{2} \neq 0$ and $\mu=0$ ):

$$
v_{E}(x)=v_{M^{4}}(x)+\Delta v_{U}(x)+\Delta v_{T}(x)+\Delta v_{M}(x),
$$

where $\Delta v_{U}(x)$ is the same as in eq. (38), $\Delta v_{T}(x)=f\left(x, 1 / 2,1 / 2, m_{T}^{2} / m_{0}^{2}, g_{U T} / \lambda\right)$ and $\Delta v_{M}(x)=f\left(x, 1 / 2,0, m_{M}^{2} / m_{0}^{2}, g_{U M} / \lambda\right)$, where the function $f\left(x, u_{1}, u_{2}, k, q\right)$ is given by

$$
\begin{aligned}
& f\left(x, u_{1}, u_{2}, k, q\right)=\frac{g}{\nu^{2}} \sum_{l_{1}, l_{2}=-\infty}^{\infty}\left[\left(\left(l_{1}-u_{1}\right)^{2}+\left(l_{2}-u_{2}\right)^{2}+\nu(k+q x)\right)^{2}\right. \\
& \quad \times \ln \frac{\left(l_{1}-u_{1}\right)^{2}+\left(l_{2}-u_{2}\right)^{2}+\nu(k+q x)}{\left(l_{1}-u_{1}\right)^{2}+\left(l_{2}-u_{2}\right)^{2}+\nu k}-x \nu q\left(\left(l_{1}-u_{1}\right)^{2}+\left(l_{2}-u_{2}\right)^{2}+\nu k\right) \\
& \left.-\frac{\nu^{3} q^{3} x^{3}}{2} \nu^{2} q^{2} x^{2}-\frac{1}{3\left(\left(l_{1}-u_{1}\right)^{2}+\left(l_{2}-u_{2}\right)^{2}+\nu k\right)}\right] .
\end{aligned}
$$

Notice that $\Delta v_{U}(x)$ is equal to $f(x, 0,0,1,1)$ but with the term with $l_{1}=l_{2}=0$ being omitted from the sum. Scaling properties of these functions, listed in Appendix C, show that the one-loop contributions have similar behaviours and varying values of $g_{U T}, g_{U M}$ and the masses amounts to rescalings only. For this reason, qualitatively the effective potential $V_{E}(\phi)$ does not change and its properties for small and large $\phi$ are the same as in the case of the periodic field only.

However, the following important observation is in order. It is seen, that in eq. (49) the leading term for $\phi \rightarrow \infty$ does not depend on the boundary conditions imposed. Actually, it may be shown that the leading behaviour of $v_{E}(x)$ for $\phi \rightarrow \infty$ is

$$
v_{E}(x) \equiv-\frac{1}{6144 \pi^{3}} \frac{\lambda_{U}}{m_{U}^{4}} \phi^{6}\left(\lambda_{U}^{3}+g_{T U}^{3}+g_{M U}^{3}\right) .
$$

Thus one finds, that due to the interactions between the different scalar fields the effective potential tends quicker to infinity than for one scalar field only. This means, that the lifetime of the vacuum state $\phi=0$, depending on the height and width of the potential barrier, is reduced due to the presence of these interactions. In order to reduce the lifetime considerably one has to choose the coupling constants large enough. However, at this point no further conclusions can be drawn, because for these values one might enter into the strong coupling regime, where we cannot trust the one-loop result. We will come back to this point in the next section, when dealing with stability in arbitrary dimensions. 


\section{Stability of the theory in arbitrary dimensions}

Let us now consider the model of Sect. 2 in the $(4+n)$ dimensional manifold $M^{4} \times T^{n}$ in order to analyze the dependence of the stability on the number $n$ of compactified dimensions. Then, instead of eq. (11) one is led to the zeta-function

$$
\zeta\left(s ; M^{4} \times T^{n}\right)=\frac{\pi^{2}}{L^{4}}\left(\frac{L}{2 \pi}\right)^{2 s} \frac{\Gamma(s-2)}{\Gamma(s)} Z_{n}^{v^{2}}\left(s-2 ; w_{1}, \ldots, w_{n}\right),
$$

with $w_{i}=\left(L / L_{i}\right)^{2}, i=1, \ldots, n$. In this general case it is still possible to determine the analytical structure of the regularized effective potential. The necessary analytical expressions are summarized in Appendix B. One finds

$$
\begin{aligned}
V_{n} V\left(\Phi^{2}\right)=-\frac{1}{4} & \frac{\pi^{2}}{L^{4}}\left\{Z_{n}^{\prime v^{2}}\left(-2 ; w_{1}, \ldots, w_{n} ; u_{1}, \ldots, u_{n}\right)\right. \\
+ & \left.Z_{n}^{v^{2}}\left(-2 ; w_{1}, \ldots, w_{n} ; u_{1}, \ldots, u_{n}\right)\left[\ln \left(\frac{L \kappa}{2 \pi}\right)^{2}+\frac{3}{2}\right]\right\},
\end{aligned}
$$

with

$$
Z_{n}^{v^{2}}\left(-2 ; w_{1}, \ldots, w_{n} ; u_{1}, \ldots, u_{n}\right)=\left\{\begin{array}{cl}
0, & \text { for } n \text { odd } \\
\frac{2(-1)^{\frac{n}{2}} \pi^{\frac{n}{2}} v^{n+4}}{\sqrt{w_{1} \ldots w_{n}}\left(\frac{n}{2}+2\right) !} & \text { for } n \text { even. }
\end{array}\right.
$$

Using (B.3) and (B.4), and the formula above, this can be written more explicitly as follows: for $n$ even

$$
\begin{gathered}
V_{n} V\left(\Phi^{2}\right)=\frac{1}{2}(-1)^{\frac{n}{2}+1} \frac{\pi^{\frac{n}{2}+2}}{\left(\frac{n}{2}+2\right) !} \frac{L_{1} \ldots L_{n}}{L^{n+4}} v^{n+4} \times \\
{\left[\frac{3}{2}+\sum_{k=3}^{\frac{n}{2}+2} \frac{1}{k}-\ln \left(\frac{\Phi}{\kappa}\right)^{2}\right]} \\
-\frac{L_{1} \ldots L_{n}}{L^{n+4}} v^{\frac{n}{2}+2} \sum_{l_{1}, \ldots, l_{n}=-\infty}^{\prime} \exp \left\{2 \pi i\left[l_{1} u_{1}+\ldots+l_{n} u_{n}\right]\right\} \times \\
{\left[\frac{l_{1}^{2}}{w_{1}}+\ldots+\frac{l_{n}^{2}}{w_{n}}\right]^{-\frac{1}{2}\left(2+\frac{n}{2}\right)} K_{\frac{n}{2}+2}\left(2 \pi v\left[\frac{l_{1}^{2}}{w_{1}}+\ldots+\frac{l_{n}^{2}}{w_{n}}\right]^{\frac{1}{2}}\right)}
\end{gathered}
$$

and for $n$ odd

$$
\begin{aligned}
& V_{n} V\left(\Phi^{2}\right)=\frac{1}{2}(-1)^{\frac{n-1}{2}} \frac{\pi^{\frac{n}{2}+3}}{\Gamma\left(\frac{n}{2}+3\right)} \frac{L_{1} \ldots L_{n}}{L^{n+4}} v^{n+4} \\
&-\frac{L_{1} \ldots L_{n}}{L^{n+4}} v^{\frac{n}{2}+2} \sum_{l_{1}, \ldots, l_{n}=-\infty}^{\infty}{ }^{\prime} \exp \left\{2 \pi i\left[l_{1} u_{1}+\ldots+l_{n} u_{n}\right]\right\} \times \\
& {\left[\frac{l_{1}^{2}}{w_{1}}+\ldots+\frac{l_{n}^{2}}{w_{n}}\right]^{-\frac{1}{2}\left(2+\frac{n}{2}\right)} K_{\frac{n}{2}+2}\left(2 \pi v\left[\frac{l_{1}^{2}}{w_{1}}+\ldots+\frac{l_{n}^{2}}{w_{n}}\right]^{\frac{1}{2}}\right) . }
\end{aligned}
$$


The leading asymptotic behaviour of the 1-loop contribution to the effective potential before renormalization is: for $n$ even

$$
V_{n} V\left(\Phi^{2}\right)=(-1)^{\frac{n}{2}} \frac{\pi^{\frac{n}{2}+2}}{2\left(\frac{n}{2}+2\right) !} \frac{L_{1} \ldots L_{n}}{L^{n+4}}\left(v^{2}\right)^{n / 2+2} \ln \frac{\lambda \phi^{2}}{2 \kappa^{2}}
$$

and for $n$ odd

$$
V_{n} V\left(\Phi^{2}\right)=(-1)^{\frac{n-1}{2}} \frac{\pi^{\frac{n}{2}+3}}{2 \Gamma\left(\frac{n}{2}+3\right)} \frac{L_{1} \ldots L_{n}}{L^{n+4}}\left(v^{2}\right)^{n / 2+2},
$$

where, as above, $v^{2}=(L \Phi)^{2} /(2 \pi)^{2}$ and $\Phi^{2}=m_{0}^{2}+\lambda \phi^{2} / 2$. As we have seen in the previous sections, in the case of the six-dimensional model the renormalization does not change the leading behaviour of the effective potential for $\phi \rightarrow \infty$. This is also true in the $(4+n)$ dimensional space-time considered here. Indeed, for $n$ even, in the renormalization conditions analogous to (15)-(18), the last one, which contains the highest derivative, would amount to adding terms proportional to $\phi^{n+4}$. This does not change the asymptotic behaviour (58). Now, let us turn to the case of $n$ odd. As is well known, for the $\lambda \phi^{4}$ theory in $(4+n)$ dimensions, due to the $Z_{2}$-symmetry, the only one-loop diagrams which are ultraviolet divergent have a number of external legs $\leq(n+3)$. Thus, for example, in five dimensions the one-loop divergences are the same as in four dimensions. In this event the renormalization conditions analogous to (15)-(18) would amount to subtraction of terms up to $\phi^{n+3}$ and hence would not affect the asymptotics (59).

Looking only at the leading behaviour we find that stability or instability of $V_{E}(\phi)$ for large $\phi$ appears always pairwise. For $k \in \mathrm{N}$, one finds instability for the dimensions $n=4 k+2,4 k+3$, and stability for $n=4 k, 4 k+1$.

Let us now include once more interactions between the scalar fields with different types of boundary conditions existing on the spacetime $M^{4} \times T^{n}$. As is well known, for different compactification lenghts $L_{i}$ there exist $2^{n}$ scalar fields on the spacetime $M^{4} \times T^{n}$. Assuming for simplicity, that their couplings to the untwisted scalar field are approximately equal, let us say $\lambda$, the leading behaviour, corresponding to eqs. (58) and (59), is multiplied by the factor $2^{n}$. Thus even for small values of the coupling constant $\lambda$, that is in the range where our results are valid, we show that for $n=4 k+2,4 k+3$, increasing dimensionality of the compact dimensions might drastically reduce the lifetime of the vacuum state $\phi=0$. This observation was our main reason for including interactions between different types of scalar fields into our analysis.

\section{Conclusions}

We have calculated in this paper the effective potential for the $\lambda \phi^{4}$-theory on a $M^{4} \times T^{2}$ space-time and showed that it is unbounded from below, namely that $V_{E}(\phi) \rightarrow-\infty$ when $\phi \rightarrow \infty$. The renormalization conditions (15)-(18) imposed guarantee that for small values of $\left(\lambda \phi^{2} L^{2}\right)$, i.e. in the weak field limit or/and for small size of the extra dimensions, the standard Coleman-Weinberg potential is recovered. This is a manifestation of the 
decoupling of heavy modes. The effect of decoupling in case of the renormalized four-point Green function in the same theory was studied in ref. [30]. We also have shown that the asymptotics of the effective potential are not affected by the renormalization procedure. We argue that it will not change either in any renormalization scheme based on adding counterterms of minimal possible powers in $\phi$ to the classical effective potential. In this sense the asymptotic behaviour of the one-loop effective potential is a renormalization scheme independent property of the theory.

The unboundness of $V_{E}(\phi)$ from below means that the vacuum $\phi=0$ for $m_{0} \neq 0$ (Fig. 1) or the non-trivial vacuum of the Coleman-Weinberg potential for $m_{0}=0$ (Fig. 3) are metastable, if the $\lambda \phi^{4}$-theory in four dimensions is the low energy limit of the theory obtained by dimensional reduction of the six-dimensional one. Of course, it is true that if the size $L$ of the two extra dimensions is small, the maximum of the potential is high enough and the value of $\phi$ where $V_{E}=0$ lies far away, so it is natural to expect that the lifetime of the vacuum is very long. However, one can estimate that in the interval where $V_{E} \leq 0$ higher loop corrections are important and may change the result drastically. Thus in the relevant range of values of $\phi$ one cannot trust the one-loop result and further analysis will be necessary. A possibility to tackle this problem would be to consider a self-interacting $O(N)$ theory and to sum over all bubble graphs dominant for large $N$ in each order, thus obtaining reliable results at larger values of $\phi$ [32].

We saw that adding the sectors of fields with antiperiodic and mixed boundary conditions on the torus $T^{2}$ and interactions between them does not change the picture qualitatively. However, we have seen that they probably reduce the lifetime of the vacuum state.

We have also extended the analysis of the asymptotics of the one-loop effective potential to the case of the spaces $M^{4} \times T^{n}$ with any $n$. The result is that in this approximation $V_{E}(\phi)$ is unstable for $n=2,3 \quad(\bmod 4)$ and is stable for $n=0,1 \quad(\bmod 4)$. When including interactions between scalar fields with different boundary conditions, our analysis suggests that for the cases $n=2,3(\bmod 4)$ contributions due to the compact space $T^{n}$ may considerably reduce the lifetime of the vacuum state $\phi=0$.

A few remarks are in order. To complete the analysis it would be important to see the effect of the change of the subtraction point $\mu^{2}$ and to calculate the $\beta$-function (similar to how it was done in ref. [14]) and the running coupling constant $\lambda(\mu)$. Then the position of the pole of the latter defines the range of validity of $V_{E}(\phi)$. In our case the running coupling constant due to contributions of heavy modes has power-like terms in $\mu^{2}$ and the position of the pole depends on $\nu$ (similarly to the case studied in [30]). These issues will be considered in a future paper.

The main messages of the result of the present analysis are the following. First, we see that if a model appears as a low-energy limit of some fundamental theory formulated on a multidimensional space-time, like the superstring in ten dimensions, then the issue of quantum stability of the vacua of this model must be re-examined, taking into account contributions of the tower of Kaluza-Klein modes. Second, if the vacuum turns out to be metastable but $\nu=\left(m_{0} L\right)^{2}$ is small enough, then the lifetime of the vacuum is expected to be rather long, in order to produce any physical effect. This may be seen as a result of the 
very different magnitudes of the scales involved, namely the inverse length $L^{-1}$ and the energy of the particles. However, at the early stage of the evolution of the Universe this might change and $L$ could be comparable to the scale factor of the 3 -space of the Universe proportional to the inverse temperature and the inverse energy scale of the particles [33]. Thus, decay of the vacuum could be essential and give rise to new effects in Kaluza-Klein cosmology. And the same could be also true for higher dimensions $n$, due to possible interactions between different types of scalar fields.

\section{Acknowledgments}

It is a pleasure to thank G. Kennedy, M. Shaposhnikov, R. Tarrach and D. Espriu for discussions and remarks that turned into an improvement of the manuscript. E.E. is gratefull to T. Muta and the whole Department of Physics, Hiroshima University for interesting discussions and warm hospitality. Yu.K. and K.K. thank the Department ECM of Barcelona University for warm hospitality. This investigation has been supported by DGICYT (Spain), project No. PB90-0022 and sabbatical grant SAB 92 0267, and by CIRIT (Generalitat de Catalunya). K.K. acknowledges financial support from the Alexander von Humboldt Foundation (Germany).

\section{A Appendix: Two dimensional Epstein-type zeta- functions}

In this appendix we want to present some results on Epstein-type zeta-functions necessary for the calculation of the effective potential of the theory considered.

As we have seen, working with two compactified dimensions, one is led to the functions

$$
Z_{2}^{v^{2}}\left(\nu ; w_{1}, w_{2} ; u_{1}, u_{2}\right)=\sum_{l_{1}, l_{2}=-\infty}^{\infty}\left[w_{1}\left(l_{1}-u_{1}\right)^{2}+w_{2}\left(l_{2}-u_{2}\right)^{2}+v^{2}\right]^{-\nu}
$$

where different values of $u_{i}$ represent different boundary conditions of the scalar field in the toroidal part $T^{2}$ of the manifold $M^{4} \times T^{2}$. In order to derive useful analytical representation of $Z_{2}^{v^{2}}$ we employ for $t \in \mathrm{R}, z \in \mathbb{C}$ the re-summation formula [31]

$$
\sum_{n=-\infty}^{\infty} \exp \left\{-t n^{2}+2 \pi i n z\right\}=\left(\frac{\pi}{t}\right) \sum_{n=-\infty}^{\infty} \exp \left\{-\frac{\pi^{2}}{t}(n-z)^{2}\right\},
$$

which is nothing else but a rewriting of the famous Jacobi theta function identity. Then one finds (for details see [4])

$$
\begin{aligned}
& Z_{2}^{v^{2}}\left(\nu ; w_{1}, w_{2} ; u_{1}, u_{2}\right)=\frac{\pi}{\sqrt{w_{1} w_{2}}} \frac{\Gamma(\nu-1)}{\Gamma(\nu)} v^{2-2 \nu} \\
& +\frac{\pi^{\nu}}{\sqrt{w_{1} w_{2}}} \frac{2}{\Gamma(\nu)} \sum_{l_{1}, l_{2}=-\infty}^{\prime} \exp \left\{2 \pi i\left[l_{1} u_{1}+l_{2} u_{2}\right]\right\} v^{1-\nu} \times
\end{aligned}
$$




$$
\left[\frac{l_{1}^{2}}{w_{1}}+\frac{l_{2}^{2}}{w_{2}}\right]^{\frac{1}{2}(\nu-1)} K_{1-\nu}\left(2 \pi v\left[\frac{l_{1}^{2}}{w_{1}}+\frac{l_{2}^{2}}{w_{2}}\right]^{\frac{1}{2}}\right)
$$

This easily gives $\left(\right.$ with $\left.w_{i}=\left(L / L_{i}\right)^{2}\right)$

$$
\begin{aligned}
& Z_{2}^{v^{2}}\left(-2 ; w_{1}, w_{2} ; u_{1}, u_{2}\right)=-\frac{\pi}{3} \frac{L_{1} L_{2}}{L^{2}} v^{6}\left[\frac{1}{3}-\ln v^{2}\right] \\
& \quad+\frac{4}{\pi^{2}} \frac{L_{1} L_{2}}{L^{2}} v^{3} \sum_{l_{1}, l_{2}=-\infty}^{\infty}{ }^{\prime} \exp \left\{2 \pi i\left[l_{1} u_{1}+l_{2} u_{2}\right]\right\}\left[\frac{l_{1}^{2}}{w_{1}}+\frac{l_{2}^{2}}{w_{2}}\right]^{-\frac{3}{2}} K_{3}\left(2 \pi v\left[\frac{l_{1}^{2}}{w_{1}}+\frac{l_{2}^{2}}{w_{2}}\right]^{\frac{1}{2}}\right),
\end{aligned}
$$

which is the result needed for the calculation of the regularized effective potential (13).

In order to see how the effective potential of Coleman and Weinberg [14] is contained in our potential, we need the small $v$ expansion of eq. (A.1). It is given by «

$$
\begin{aligned}
Z_{2}^{v^{2}}\left(\nu ; w_{1}, w_{2} ; u_{1}, u_{2}\right)= & {\left[w_{1} u_{1}^{2}+w_{2} u_{2}^{2}+v^{2}\right]^{-\nu} } \\
& +\sum_{j=0}^{\infty}(-1)^{j} \frac{\Gamma(s+j)}{j ! \Gamma(s)} Z_{2}\left(s+j ; w_{1}, w_{2} ; u_{1}, u_{2}\right) v^{2 j}
\end{aligned}
$$

with

$$
Z_{2}\left(\nu ; w_{1}, w_{2} ; u_{1}, u_{2}\right) \sum_{l_{1}, l_{2}=-\infty}^{\infty} '\left[w_{1}\left(l_{1}-u_{1}\right)^{2}+w_{2}\left(l_{2}-u_{2}\right)^{2}\right]^{-\nu}
$$

This yields

$$
\begin{aligned}
& Z_{2}^{\prime v^{2}}\left(-2 ; w_{1}, w_{2} ; u_{1}, u_{2}\right)=-\frac{1}{2}\left[w_{1} u_{1}^{2}+w_{2} u_{2}^{2}+v^{2}\right]^{2} \ln \left(w_{1} u_{1}^{2}+w_{2} u_{2}^{2}+v^{2}\right) \\
& \quad+\frac{1}{2} Z_{2}^{\prime}\left(-2 ; w_{1}, w_{2} ; u_{1}, u_{2}\right)+v^{2}\left[\frac{1}{2}\left[w_{1} u_{1}^{2}+w_{2} u_{2}^{2}\right]+Z_{2}^{\prime}\left(-1 ; w_{1}, w_{2} ; u_{1}, u_{2}\right)\right] \\
& \quad+\frac{1}{2} v^{4}\left[\frac{3}{2}+Z_{2}^{\prime}\left(0 ; w_{1}, w_{2} ; u_{1}, u_{2}\right)\right]-\frac{1}{6} v^{6} P P Z_{2}\left(1 ; w_{1}, w_{2} ; u_{1}, u_{2}\right) \\
& \quad+\sum_{j=2}^{\infty}(-1)^{j} \frac{(j-1) !}{(j+2) !} v^{2 j+4} Z_{2}\left(j ; w_{1}, w_{2} ; u_{1}, u_{2}\right),
\end{aligned}
$$

with $P P Z_{2}\left(1 ; w_{1}, w_{2} ; u_{1}, u_{2}\right)$ denoting the finite part of $Z_{2}\left(1 ; w_{1}, w_{2} ; u_{1}, u_{2}\right)$. Eq. (A.7) is the result needed for the derivation of (40).

\section{B Appendix: Epstein-type zeta-functions in arbitrary dimensions}

Compactifying $n$ dimensions, one is naturally led to the functions

$$
Z_{n}^{v^{2}}\left(\nu ; w_{1}, \ldots, w_{n} ; u_{1}, \ldots, u_{n}\right)=\sum_{l_{1}, \ldots, l_{n}=-\infty}^{\infty}\left[w_{1}\left(l_{1}-u_{1}\right)^{2}+\ldots+w_{n}\left(l_{n}-u_{n}\right)^{2}+v^{2}\right]^{-\nu}
$$


Here the analytical continuation reads

$$
\begin{gathered}
Z_{n}^{v^{2}}\left(\nu ; w_{1}, \ldots, w_{n} ; u_{1}, \ldots, u_{n}\right)=\frac{\pi^{\frac{n}{2}}}{\sqrt{w_{1} \ldots w_{n}}} \frac{\Gamma\left(\nu-\frac{n}{2}\right)}{\Gamma(\nu)} v^{n-2 \nu} \\
+\frac{\pi^{\nu}}{\sqrt{w_{1} \ldots w_{n}}} \frac{2}{\Gamma(\nu)} \sum_{l_{1}, \ldots, l_{n}=-\infty}^{\infty} \exp \left\{2 \pi i\left[l_{1} u_{1}+\ldots+l_{n} u_{n}\right]\right\} v^{\frac{n}{2}-\nu} \times \\
{\left[\frac{l_{1}^{2}}{w_{1}}+\ldots+\frac{l_{n}^{2}}{w_{n}}\right]^{\frac{1}{2}\left(\nu-\frac{n}{2}\right)} K_{\frac{n}{2}-\nu}\left(2 \pi v\left[\frac{l_{1}^{2}}{w_{1}}+\ldots+\frac{l_{n}^{2}}{w_{n}}\right]^{\frac{1}{2}}\right) .}
\end{gathered}
$$

Thus we find the quantities relevant for the effective potential. For $n$ even, we have $\left(w=\left(L / L_{i}\right)^{2}\right)$

$$
\begin{gathered}
Z_{n}^{\prime v^{2}}\left(-2 ; w_{1}, \ldots, w_{n} ; u_{1}, \ldots, u_{n}\right)=\frac{2(-1)^{\frac{n}{2}}}{\left(\frac{n}{2}+2\right) !} \frac{\pi^{\frac{n}{2}} L_{1} \ldots L_{n}}{L^{n}} v^{n+4}\left[\psi\left(\frac{n}{2}+3\right)-\psi(3)-\ln v^{2}\right] \\
+\frac{4}{\pi^{2}} \frac{L_{1} \ldots L_{n}}{L^{n}} v^{\frac{n}{2}+2} \sum_{l_{1}, \ldots, l_{n}=-\infty}^{\infty} \exp \left\{2 \pi i\left[l_{1} u_{1}+\ldots+l_{n} u_{n}\right]\right\} \times \\
{\left[\frac{l_{1}^{2}}{w_{1}}+\ldots+\frac{l_{n}^{2}}{w_{n}}\right]^{-\frac{1}{2}\left(2+\frac{n}{2}\right)} K_{\frac{n}{2}+2}\left(2 \pi v\left[\frac{l_{1}^{2}}{w_{1}}+\ldots+\frac{l_{n}^{2}}{w_{n}}\right]^{\frac{1}{2}}\right)}
\end{gathered}
$$

whereas for $n$ odd we find

$$
\begin{gathered}
Z_{n}^{\prime v^{2}}\left(-2 ; w_{1}, \ldots, w_{n} ; u_{1}, \ldots, u_{n}\right)=(-1)^{\frac{n+1}{2}} \frac{2 \pi^{\frac{n}{2}+1} L_{1} \ldots L_{n}}{\Gamma\left(\frac{n}{2}+3\right) L^{n}} v^{n+4} \\
+\frac{4}{\pi^{2}} \frac{L_{1} \ldots L_{n}}{L^{n}} v^{\frac{n}{2}+2} \sum_{l_{1}, \ldots, l_{n}=-\infty}^{\infty} \exp \left\{2 \pi i\left[l_{1} u_{1}+\ldots+l_{n} u_{n}\right]\right\} \times \\
{\left[\frac{l_{1}^{2}}{w_{1}}+\ldots+\frac{l_{n}^{2}}{w_{n}}\right]^{-\frac{1}{2}\left(2+\frac{n}{2}\right)} K_{\frac{n}{2}+2}\left(2 \pi v\left[\frac{l_{1}^{2}}{w_{1}}+\ldots+\frac{l_{n}^{2}}{w_{n}}\right]^{\frac{1}{2}}\right) .}
\end{gathered}
$$

Eq. (B.3) and (B.4) are the needed results to analyze the large $\phi$ behaviour in the selfinteracting theory in a spacetime of the form $M^{4} \times T^{n}$.

\section{Appendix: One-loop contributions to the effective potential}

For $m_{0} \neq 0$ the terms $\Delta v_{2}(x)$ and $\Delta v_{3}(x)$ in eq. (36) are

$$
\begin{aligned}
\Delta v_{2}(x) & =\frac{g x^{2}}{2} \sum_{l_{1}, l_{2}=-\infty}^{\infty}\left\{-2 \ln \frac{N^{2}\left(l_{i}\right)+\nu(1+y)}{N^{2}\left(l_{i}\right)+\nu}+\frac{2 y \nu}{N^{2}\left(l_{i}\right)+\nu(1+y)}\right. \\
& \left.-\frac{172}{3} \frac{y^{2} \nu^{2}}{\left[N^{2}\left(l_{i}\right)+\nu(1+y)\right]^{2}}+\frac{80 y^{3} \nu^{3}}{\left[N^{2}\left(l_{i}\right)+\nu(1+y)\right]^{3}}-\frac{32 y^{4} \nu^{4}}{\left[N^{2}\left(l_{i}\right)+\nu(1+y)\right]^{4}}\right\}
\end{aligned}
$$


and

$$
\begin{aligned}
\Delta v_{3}(x) & =\frac{2 g \nu x^{3}}{3} \sum_{l_{1}, l_{2}=-\infty}^{\infty}\left\{\frac{1}{N^{2}\left(l_{i}\right)+\nu}-\frac{1}{N^{2}\left(l_{i}\right)+\nu(1+y)}\right. \\
& \left.+\frac{6 y \nu}{\left[N^{2}\left(l_{i}\right)+\nu(1+y)\right]^{2}}-\frac{8 y^{2} \nu^{2}}{\left[N^{2}\left(l_{i}\right)+\nu(1+y)\right]^{2}}+\frac{16}{5} \frac{y^{3} \nu^{3}}{\left[N^{2}\left(l_{i}\right)+\nu(1+y)\right]^{4}}\right\},
\end{aligned}
$$

where

$$
N^{2}\left(l_{i}\right)=l_{1}^{2} w_{1}+l_{2}^{2} w_{2} .
$$

For $m_{0}=0$ the quantum corrections in eq. (43) are equal to:

$$
\begin{aligned}
\Delta u_{1}(z) & =6 g \sum_{l_{1}, l_{2}=-\infty}^{\infty},\left\{\frac{\left[N^{2}\left(l_{i}\right)+z t\right]^{2}}{t^{2}} \ln \frac{N^{2}\left(l_{i}\right)+z t}{N^{2}\left(l_{i}\right)}-\frac{z}{t} N^{2}\left(l_{i}\right)\right. \\
& \left.-\frac{3}{2} z^{2}-\frac{1}{3} \frac{z^{3} t}{N^{2}\left(l_{i}\right)}\right\} \\
\Delta u_{2}(z) & =3 g z^{2} \sum_{l_{1}, l_{2}=-\infty}^{\infty},\left\{-2 \ln \frac{N^{2}\left(l_{i}\right)+t}{N^{2}\left(l_{i}\right)}+\frac{2 t}{N^{2}\left(l_{i}\right)+t}-\frac{172}{3} \frac{t^{2}}{\left[N^{2}\left(l_{i}\right)+t\right]^{2}}\right. \\
& \left.+\frac{80 t^{3}}{\left[N^{2}\left(l_{i}\right)+t\right]^{3}}-\frac{32 t^{4}}{\left[N^{2}\left(l_{i}\right)+t\right]^{4}}\right\}, \\
\Delta u_{3}(z) & =12 g t^{2} z^{3} \sum^{\infty},\left\{\frac{2}{N^{2}\left(l_{i}\right)\left(N^{2}\left(l_{i}\right)+t\right)}+\frac{6}{\left[N^{2}\left(l_{i}\right)+t\right]^{2}}\right. \\
& \left.-\frac{8 t}{\left[N^{2}\left(l_{i}\right)+t\right]^{3}}+\frac{16}{5} \frac{t^{2}}{\left[N^{2}\left(l_{i}\right)+t\right]^{4}}\right\},
\end{aligned}
$$

where $N^{2}\left(l_{i}\right)$ is given by eq. (C.3).

The function $f\left(x, \nu, u_{1}, u_{2}, k, q\right)$ defined in eq. (51) (here we indicate its dependence on $\nu$ explicitly), which characterizes one-loop contribution of the heavy modes with arbitrary boundary conditions, posesses the following scaling properties:

$$
\begin{gathered}
f\left(x, \nu, u_{1}, u_{2}, k, \lambda q\right)=f\left(\lambda x, \nu, u_{1}, u_{2}, k, q\right), \\
f\left(x, \nu, u_{1}, u_{2}, \lambda k, q\right)=\lambda^{2} f\left(x / \lambda, \lambda \nu, u_{1}, u_{2}, k, q\right) .
\end{gathered}
$$

These scaling properties have been used to show explicitly that interactions between the different types of scalar fields (see section 4) do not qualitatively change the effective potential. 


\section{References}

[1] G.W. Gibbons, J. Phys. A11 (1978) 1341.

E.J. Copeland and D.J. Toms, Nucl. Phys. B255 (1985) 201.

G.R. Shore, Ann. Phys. 128 (1980) 376.

G. Kennedy, Phys. Rev. D23 (1981) 2884.

D.J. Toms, Phys. Rev. D25 (1982) 2536.

Yu.P. Goncharov, Phys. Lett. 119B (1982) 403.

J. Ambjorn and S. Wolfram, Ann. Phys. 147 (1983) 1.

J.S. Dowker, Class. Quant. Grav. 1 (1984) 359.

A. Actor, Class. Quant. Grav. 7 (1990) 1463.

G. Cognola, K. Kirsten and S. Zerbini, Phys. Rev. D48 (1993) 790.

[2] E. Elizalde and K. Kirsten, J. Math. Phys. 35 (1994) 1260.

[3] E. Elizalde and A. Romeo, Phys. Lett. B244 (1990) 387.

G. Denardo and E. Spallucci, Nuovo Cimento A64 (1981) 27.

G. Denardo and E. Spallucci, Nucl. Phys. B169 (1980) 514.

K. Kirsten, Class. Quantum Grav. 11 (1994) 57.

A.A. Bytsenko, G. Cognola, L. Vanzo and S. Zerbini, Quantum Fields and Extended Objects in Space-Times with Constant Curvature Spatial Section, Preprint Trento, U.T.F. 325.

[4] K. Kirsten, J. Phys. A26 (1993) 2421.

[5] H.B.G. Casimir, Proc. Kon. Ned. Akad. Wet. 51 (1948) 793.

S.K. Blau, M. Visser and A. Wipf, Nucl. Phys. B310 (1988) 163.

E. Elizalde, Nuovo Cimento B104 (1989) 685.

G. Cognola, L. Vanzo and S. Zerbini, J. Math. Phys. 33 (1992) 222.

F. Caruso, N.P. Neto, B.F. Svaiter and N.F. Svaiter, Phys. Rev. D43 (1991) 1300.

B.F. Svaiter and N.F. Svaiter, J. Math. Phys. 32 (1991) 175.

L.H. Ford, Phys. Rev. D21 (1980) 933.

D.J. Toms, Phys. Rev. D21 (1980) 2805.

D.J. Toms, Phys. Rev. D21 (1980) 928.

K. Kirsten, J. Phys. A A24 (1991) 3281.

K. Kirsten, Class. Quantum Grav. 8 (1991) 2239.

J.S. Dowker and J.P. Schofield, Nucl. Phys. B327 (1989) 267. J.S. Dowker and R. Banach, J. Phys. A11 (1978) 2255.

P. Candelas and S. Weinberg, Nucl. Phys. B237 (1984) 397.

B.P. Dolan and C. Nash, Commun. Math. Phys. 148 (1992) 139.

[6] G. Plunien, Müller B and W. Greiner, Phys. Rep. 134 (1986) 87.

[7] A. Salam and J. Strathdee, Ann. Phys. 141 (1982) 316.

[8] M.J. Duff, B.E.W. Nilsson and C.N. Pope, Phys. Rep. C 130 (1986) 1. 
[9] N.S. Manton, Nucl. Phys. B 158 (1979) 141.

[10] Yu.A. Kubyshin, J.M. Mourão, G. Rudolph and I.P. Volobujev, Dimensional Reduction of Gauge Theories, Spontaneous Compactification and Model Building, Lecture Notes in Physics, Vol. 349 (Springer-Verlag, Berlin, 1989).

[11] D. Kapetanakis and G. Zoupanos, Phys. Rep. C219 (1992) 1.

[12] T. Appelquist and A. Chodos, Phys. Rev. D28 (1983) 772.

E.S. Fradkin and A.A. Tseytlin, Nucl. Phys. B227 (1983) 252.

P. Howe, P.K. Townsend and K. Stelle, Nucl. Phys. B 214 (1983) 519.

S. Mandelstam, Nucl. Phys. B 213 (1983) 149.

N. Marcus and A. Sagnotti Nucl. Phys. B256 (1985) 77.

R. Coquereux and G. Esposito-Farese, Class. Quant. Grav. 7 (1990) 1583.

[13] R.H. Brandenberger, Rev. Mod. Phys. 57 (1985) 1.

[14] S. Coleman and E. Weinberg, Phys. Rev. D7 (1973)1888.

[15] A.P. Demichev, Yu.A. Kubyshin and J.I. Pérez Cadenas, Phys. Lett. B323 (1994) 139.

E. Elizalde and Yu. Kubyshin. Possible evidences of Kaluza-Klein particles in a scalar model with spherical compactification. Preprint UAB-ECM-PF 94/4, University of Barcelona (1994). To appear in J. Phys. A.

[16] E.W. Kolb and R. Slansky, Phys. Lett. 135B

[17] V.S. Kaplunovsky, Nucl. Phys. B307 (1988) 145.

L.J. Dixon, V.S. Kaplunovsky and J. Louis, Nucl. Phys. B355 (1991) 649.

I. Antoniadis, Phys. Lett. B246 (1990) 377.

[18] I. Antoniadis and K. Benakli, Phys. Lett. B 326 (1994) 69.

I. Antoniadis, K. Benakli and M. Quirós, Phys. Lett. B331 (1994) 313.

[19] C.W. Bernard, Phys. Rev. D9 (1974) 3312.

L. Dolan and R. Jackiw, Phys. Rev. D9 (1974) 3320.

N.P. Landsman and Ch.G. von Weert, Phys. Rep. 145 (1987) 141.

D.J. Gross, R.D. Pisarski and S. Rudaz, Rev. Mod. Phys. 53 (1981) 43.

J.I. Kapusta, Finite temperature field theory (Cambridge University, Cambridge, 1989).

[20] T. Appelquist and A. Chodos, Phys. Rev. Lett. 50 (1983) 141.

[21] T. Appelquist, A. Chodos and E. Myers, Phys. Lett. 127B (1983) 51.

[22] M. Rubin and B. Roth, Nucl. Phys. B226 (1983) 444.

[23] M. Rubin and B. Roth, Phys. Lett. B127 (1983) 55. 
[24] S.K. Blau, E.I. Guendelman, A. Taormina and L.C.R. Wijewardhana, Phys. Lett. B144 (1984) 30.

[25] S. Weinberg, Phys. Rev. D 7 (1973) 2887.

R. Jackiw, Phys. Rev. D 9 (1974) 1686.

S. Weinberg, Phys. Lett. B 91 (1980) 51.

[26] A. Actor, Class. Quantum Grav. 5 (1988) 1415.

[27] J.E. Bagnasco and M. Dine, Phys. Lett. B 303 (1993) 308.

P. Arnold and O. Espinosa, Phys. Rev. D 47 (1993) 3546.

P. Arnold and L. Yaffe, Seattle preprint UW/PT-93-24 (1993).

A. Mebecker, Z. Phys. C 60 (1993) 271.

K. Farakos, K. Kajante, K. Rummukainen and M. Shaposhnikov, CERN preprint, CERN-TH.6973/94 (1994).

[28] R. Critchley and J.S. Dowker, Phys. Rev. D 13 (1976) 3224.

S.W. Hawking, Commun. Math. Phys. 55 (1977) 133.

[29] D.J. Toms, Ann. Phys. 129 (1980) 334.

[30] Yu. Kubyshin, D. O'Connor and C.R. Stephens, Class. Quant. Grav. 10 (1993) 2519. Yu. Kubyshin, D. O'Connor and C.R. Stephens, in: "Quarks '92". Proceedings of the International Seminar (May 1992, Zvenigorod, Russia). Eds. D.Yu. Grigoriev, V.A. Matveev, V.A. Rubakov and P.G. Tinyakov (World Scientific, Singapore, 1993), p. 359.

[31] E. Hille, Analytic Function Theory vol II, Ginn, Boston, (1962).

[32] K.G. Wilson, Phys. Rev. D 7 (1973) 2911.

S. Coleman, R. Jackiw and H.D. Politzer, Phys. Rev. D 10 (1974) 2491.

H.J. Schnitzer, Phys. Rev. D 10 (1974) 1800.

[33] For review see E.W. Kolb and M.S. Turner, The Early Universe. (Addison-Wesley Publishing Co., 1990). 


\section{Figure captions}

Fig. 1 Plots of the functions $v_{M^{4}}(x)$ (labelled by CW) and $v_{E}(x)$ for the case of a massive field $\phi$ with periodic boundary conditions; $y=0, \xi=0$ and $w_{1}=w_{2}=1$ for various $\nu$. The number near the curve corresponds to the value of $\nu$.

Fig. 2 Plots of the functions $u_{M^{4}}(z)$ (labelled by CW) and $u_{E}(z)$ for the case of a massless field $\phi$ with periodic boundary conditions; $\eta=0$ and $w_{1}=w_{2}=1$. The number near the curve corresponds to the value of $t$.

Fig. 3 The same plots as in Fig. 2 but for the interval $0<z<50$. 
This figure "fig1-1.png" is available in "png" format from: http://arxiv.org/ps/hep-th/9410101v1 
This figure "fig1-2.png" is available in "png" format from: http://arxiv.org/ps/hep-th/9410101v1 
This figure "fig1-3.png" is available in "png" format from: http://arxiv.org/ps/hep-th/9410101v1 